\title{
Instrumental Resolution Limit By Magnetic Thermal Noise From Conductive Parts
}

\author{
Stephan Uhlemann, Heiko Müller, Peter Hartel, Joachim Zach and Max. Haider
}

CEOS Corrected Electron Optical Systems, Heidelberg, Germany.

Since recently the focus spread during the acquisition of high-resolution TEM images can be reduced by the correction of the chromatic aberration. It is known that the contrast transfer is less improved than expected [1]. Instead, an alternative contrast damping mechanism of the form $\exp \left[-2(\pi \sigma|g|)^{2}\right]$ now limits the information transfer of larger spatial frequencies $g$, which was identified as an isotropic image spread $\sigma$ of several ten picometers [1]. After testing a lot of hypotheses the origin of this contrast dampening could tentatively be identified as a magnetic dipole noise emitted from the conductive solid materials of the parts around the beam path in the microscope. It is known from various applications of the fluctuation-dissipation theorem (e.g. [2]) that such noise exists. However, it is surprising that both, conductive non-magnetic and magnetic materials, contain microscopic currents up to such high frequencies that the integral noise becomes relevant in electron microscopy. First, we report on our fundamental experiments which show that different materials at room temperature cause image spread. Second, we show experimentally that the variance of the magnetic noise flux induced image spread is proportional to $k_{B} T$. Third, we describe progress towards developing and testing methods how to calculate the amount of noise emitted from a given geometry and how the electrons are affected.

Except for a few lenses we completely removed the CsCc-corrector from a Titan instrument to provide sufficient space for the experiments. Figure 1 shows the remaining setup of the ST objective lens followed by a few transfer/adapter lenses which image into the SA plane about $970 \mathrm{~mm}$ below specimen. The free space within the column contains $\mu$-metal screens $(\varnothing 10 \mathrm{~cm}$, not shown), the vacuum tube $\varnothing 6 \mathrm{~cm}$ and a $\varnothing 15 \mathrm{~mm} \mathrm{LN}_{2}$ coolable copper tube which accommodates the test tubes, see Figure 2. In order to be able to quantify the noise amplitude with errors $<10 \%$, we employed unusual long tubes $(\mathrm{l}=0.5 \mathrm{~m})$ with about $3 \mathrm{~mm}$ bores. The beam diameter of the relevant $50 \mathrm{mrad}$ scattered electrons was about 10 times smaller, i.e. $200-400 \mu \mathrm{m}$, and a single side-band required half of the latter space. The covariance of the noise flux roughly can be characterized by the largest correlation length $\xi$ of an axis interval $\Delta z$ where the expectation of $\langle B(z) \cdot B(z+\Delta z)$ - is non-zero. Actually the product of this length and the variance of the flux density $\xi \cdot \operatorname{var}(B)$ is responsible for the electron deflection and can therefore be measured.

The FFTs in Figure 3 exemplarily show the dampening of the single side-band contrast at the achromatic rings resulting from $2 \mathrm{deg}$ illumination tilt at $80 \mathrm{kV}$. The method of evaluating the contrast along the rings quantitatively is presented elsewhere [3]. Figure 4 shows the temperature dependence $[\xi \cdot \operatorname{var}(B)](T)$ deduced from the observed additional image spread of the tubes $\sigma^{2}=\left(e / 2 \mathrm{~m}_{0} U^{*}\right) \cdot(M f)^{2} l \cdot \xi \operatorname{var}(B)$. Here the three experiments (B1)-(B3) are referred to a null experiment (A) which collects the smallest amount of image spread, since the aperture is demagnified just behind the objective lens. We take the fact that the three measurements arrange along a straight line - if (B1) is placed at $0 \mathrm{~K}$ - as an indication, that the stainless steel tube would become "invisible" (noise free) if it could be cooled down to absolute zero. The offset however is to be interpreted as a different noise contribution of the warm parts before and after the cooled area if the beam setup (B) is used instead of (A).

The findings of this work provide new constraints to the design of electron optical instruments. Defining two dimensionless constants for geometry and material we provisionally suggest a relation to calculate 
the noise amplitude. If $a$ is the typical radius, $c_{g} \approx 0.2$ (cylinder), $c_{m}=1$ for thin conductors and larger (3...4) for magnets we write $\xi \operatorname{var}(B)=c_{g} c_{m} \cdot \mu_{0} k_{B} T / a^{2}$.

\section{References:}

[1] M. Haider et al, Microsc.Microanal. 16 (2010) p. 393.

[2] S.-K. Lee and M.V. Romalis, J.Appl.Phys. 103 (2008) p. 084904.

[3] H. Müller et al, This Conference, M\&M (2013), poster session A05.

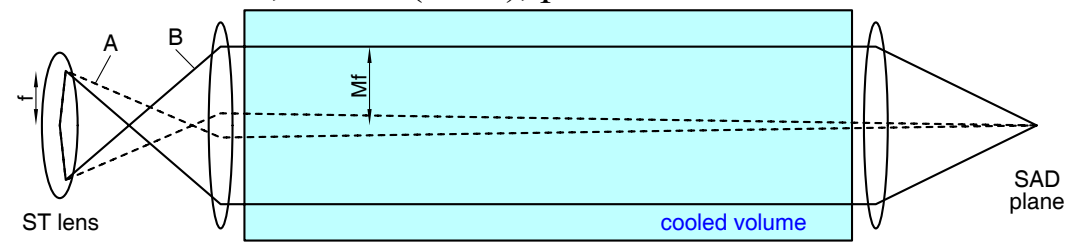

Figure 1. Beam setup of the experiments A and B. The aperture magnification $\mathrm{M}$ is indicated.

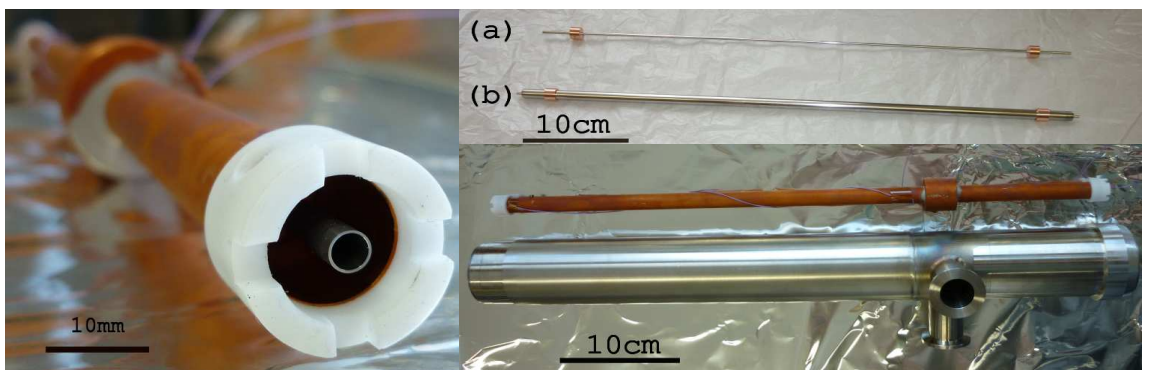

Figure 2. Thin stainless steel liner tube within copper cooler (left). Two $1=516 \mathrm{~mm}$ long samples: Ø3mm liner tube (a), and a stack of $\mu$-metal tubes (b). Vacuum tube with feed through to $\mathrm{LN}_{2}$ reservoir (right).
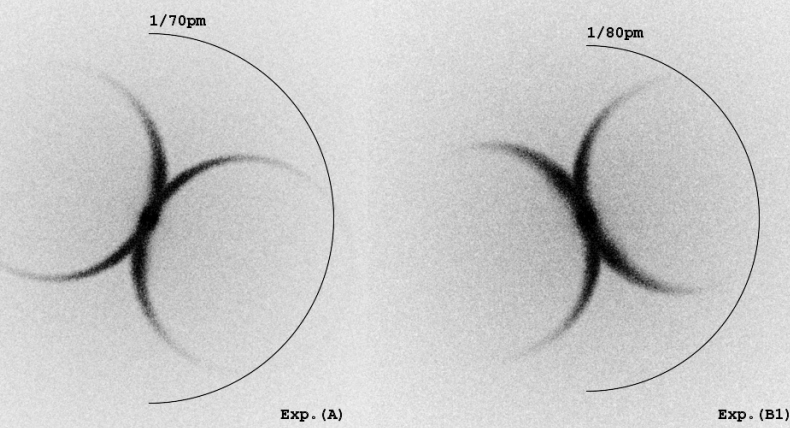

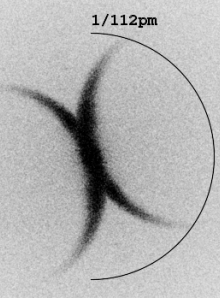

Exp.(B2)

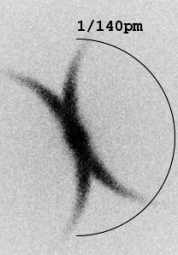

Exp. (B3)

Figure 3. Achromatic ring diffractograms obtained from $2 \mathrm{~nm}$ amorphous tungsten films. (A) Null experiment, (B1) empty cooler, (B2) liner tube (a) at $\mathrm{T} \approx 120 \mathrm{~K}$, and (B3) liner tube (a) at $\mathrm{T} \approx 300 \mathrm{~K}$.

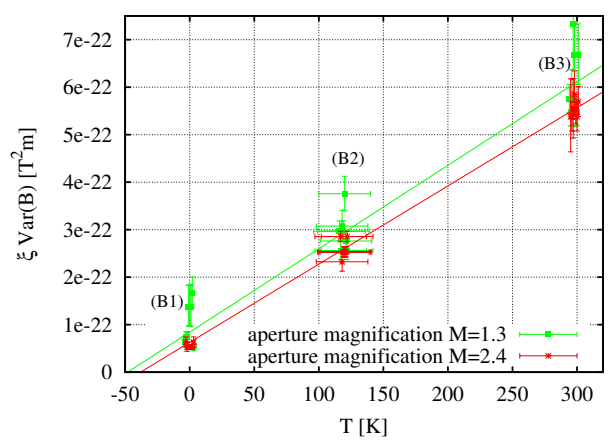

Figure 4. Two liner tube experiments with different aperture magnifications. The deduced variance of the magnetic noise flux density times correlation length $\xi$ is depicted. 